\title{
EL PENSAMIENTO COMPUTACIONAL PARA POTENCIAR EL DESARROLLO DE HABILIDADES RELACIONADAS CON LA RESOLUCIÓN CREATIVA DE PROBLEMAS
}

\section{THE COMPUTATIONAL THINKING ABOUT PROMOTING DEVELOPMENT OF SKILLS RELATED CREATIVE SOLVING PROBLEMS}

Mauricio Pérez Palencia ${ }^{1}$

1. Licenciado en informática y medios audiovisuales por la Universidad de Córdoba, Colombia. Docente de educación básica y media. Maestrante en Educación. Sistema de Universidades Estatales del Caribe (SUE Caribe) (Colombia). E-Mail: mperezyp@gmail.com

Citación sugerida:

Pérez Palencia, M. (2017). El pensamiento computacional para potenciar el desarrollo de habilidades relacionadas con la resolución creativa de problemas. 3C TIC: Cuadernos de desarrollo aplicados a las TIC, 6(1), 38-63. DOI: <http://dx.doi.org/10.17993/3ctic.2017.55.38-63/>. 


\section{RESUMEN}

El presente documento es un aporte científico a la didáctica de las tecnologías de la información y la comunicación, desde el cual se evidencian las potencialidades y privilegios pedagógicos que ofrecen las ciencias de la computación para el desarrollo de competencias relacionadas con la resolución creativa de problemas. Se lleva a cabo a través de un estudio cuasi experimental y desde una propuesta metodológica fundada en la programación de computadoras mediante el lenguaje de programación visual "Scratch" como principal herramienta pedagógica mediacional.

\section{ABSTRACT}

This document is a scientific contribution to the teaching of information and communications technologies, from which the potential and educational privileges offered by computer science for the development of skills related to creative solving problems are evident. It is developed through a quasi-experimental study from a methodological proposal based on computer programming through visual programming language "Scratch" mediational main teaching tool.

\section{PALABRAS CLAVE}

Pensamiento Computacional, Programación de Computadoras, Resolución de problemas, Scratch, Creatividad.

\section{KEY WORDS}

Computational Thinking, Computer programming, Problem Solving, Scratch, Creativity. 


\section{INTRODUCCIÓN}

Hoy en día son muchos los mecanismos, metodologías, y herramientas destinadas y/o adaptadas como apoyo a la educación, pero en esta atmósfera emergente mediacional, y durante la última década ha resurgido la programación de computadoras y el pensamiento computacional como fuentes sustanciales para propiciar escenarios pedagógicos de alta productividad pedagógica en ambientes de educación escolarizada. Estas temáticas, debido a su complejidad conceptual y exigencias cognitivas, se asocian al campo específico de las ingenierías y a disposición de personal experimentado en el campo de las ciencias de la computación. Esta concepción segmentaria comenzó a dar un giro positivo hacia la pedagogía, y es en los inicios de los años 70 cuando se da el primer asomo significativo del uso educativo de programación. Esta propuesta disruptiva fue implementada por Papert con su lenguaje de programación Logo y replicada en varios escenarios educativos en el mundo, y se ha vuelto fuerte debido a los nuevos lenguajes de programación visuales, como Alicia, Kodu, Scratch, y permitiéndole a los jóvenes estudiantes a las aplicaciones del programa sin la necesidad de aprender la compleja sintaxis de los lenguajes de programación tradicionales (Moreno-León, 2015).

Esta concepción del desarrollo de habilidades mentales de considerado nivel potenciadas desde campos como la programación de computadoras, es lo que motiva a Wing (2006, p.33), principal precursora del pensamiento computacional, a considerar que este "consiste en la resolución de problemas, el diseño de sistemas, y la comprensión de la conducta humana haciendo uso de los conceptos fundamentales de la informática". No obstante, el carácter sugestivo inducido por la aparente rigurosidad que suponen estas temáticas no constituye factores adversos para el aprovechamiento del potencial didáctico de estos conceptos y técnicas propias de la ingeniería de software. Al respecto, Wing (2006, p.33), asevera que "El pensamiento computacional es una habilidad fundamental para todos, no sólo para los informáticos. Para la lectura, escritura y aritmética, hay que añadir pensamiento computacional a la capacidad analítica de cada niño".

De manera que, la presente es una propuesta sustentada en el potencial pedagógico de la programación de computadoras, asumida ésta como principal factor técnico-pedagógico para el desarrollo de habilidades computacionales, por medio de la cual se pretende demostrar que el desarrollo del pensamiento computacional potencia habilidades relacionadas con la resolución creativa de problemas. Para este propósito se toma en cuenta como principal herramienta mediacional el lenguaje de programación Scratch, el cual es un entorno de programación visual (por bloques) diseñado para que niños mayores de 6 años desarrollen habilidades de pensamiento computacional, aprovechando con ello el carácter interdisciplinario de las ciencias de la computación desde la perspectiva de la programación de computadoras (Resnick et al., 2009).

Uno de los principales objetivos de Scratch como entorno didáctico de programación es precisamente el desarrollo de otras habilidades y mejorar el aprendizaje de otras disciplinas mientras se aprende o se ponen en funcionamiento los conceptos de programación (Resnick, 
2013). Esto se lleva a cabo mediante el abordaje de situaciones problema implementando el modelo de solución de problemas de Polya (1945), para quien, cuando se resuelven problemas intervienen cuatro operaciones mentales: Entender el problema, Trazar un plan, Ejecutar el plan (resolver) y Revisar. Esta propuesta teórica coincide conceptualmente con modelos de resolución de problemas por computadora, entre los que se destaca el referenciado por Joyanes (1996), quien desde un plano concreto describe una metodología que implica un análisis, el diseño de una solución, su puesta en marcha y su verificación del algoritmo.

En concordancia con esta idea, Newell y Simons (1972), sugieren que la resolución de problemas puede ser modelada por una máquina procesadora de información (computadoras), es decir, promover la creatividad de los estudiantes y demostrar el vínculo entre la teoría computacional y las aplicaciones en el mundo real. Es necesario enfatizar entonces en la construcción de modelos propuestos por los estudiantes al afrontar una situación problema, cuyo proceso de resolución puede representar solo una de las diversas maneras de hallar una solución. Este hecho sugiere que cada problema puede tener varias formas de resolverlo, en esta fuente de analítica y creativa radica la fuente de riqueza para el desarrollo tanto de la inteligencia como de la creatividad, al mismo tiempo que se aprenden conceptos y sus aplicaciones Rugarcia y Delgado (1987) citando a Whimbey (1975) y Raanheim (1974).

Por otra parte, dentro del proceso de investigación también se tiene en cuenta otro aspecto interesante a resaltar: el constructo Creatividad, concebida no como estructura, sino como proceso, según los aportes de (García, 1998), sentido en el cual cobra coherencia con la naturaleza procesual de los demás soportes teóricos de esta investigación.

Lo expuesto hasta aquí representa un viaje científico del cual se extraen los principales apartes y aspectos, los cuales se refieren primeramente a aspectos metodológicos, desde lo investigativo y lo didáctico, en segundo lugar, a los resultados obtenidos y sus implicaciones en el estudio, en tercer lugar, las consideraciones discursivas en torno a la discusión reflexiva y crítica, y, por último, según lo planteado en los propósitos, a abordar los aspectos referidos a las conclusiones obtenidas con la investigación.

\section{METODOLOGÍA}

\subsection{CONTEXTUALIZACIÓN}

La presente investigación se llevó a cabo en las instituciones educativas Rafael Núñez y Luis Villafañe Pareja, de los corregimientos San Andrés y Martín Alonso, del Municipio de Córdoba, departamento de Bolívar. La primera de ellas la posee un total de 570 estudiantes y la segunda 576, en los distintos niveles de preescolar, básica y media. Estas instituciones en los últimos años se perfilaron hacia la formación técnica, el caso de la I.E Rafael Núñez forma desde el 2012 bachilleres con énfasis técnico en sistemas, en acompañamiento logístico y 
pedagógico con el Servicio Nacional de Aprendizaje SENA. Solo en 2015, sus egresados obtuvieron el título de Técnico en Sistemas emanado autónomamente por la institución, independientemente del acompañamiento del Sena. Situación similar atañe a Luis Villafañe Pareja, la cual forma con acompañamiento del Sena, bachilleres técnicos en Producción en procesamiento de frutas y hortalizas.

El contexto en el que se enmarcan ambas poblaciones, las cuales distan entre sí por $9 \mathrm{~km}$ a través de vías secundarias y terciarias, está marcado socialmente por problemas de orden público en el sentido de las violentas perturbaciones provocadas por grupos ilegales alzados en armas, especialmente en la década de los 90 . Tal situación es la génesis de problemáticas sociales en las que se destacan el desplazamiento forzado ( $35 \%$ de familias desplazadas, SISBEN) y la desintegración del núcleo familiar.

Dadas las condiciones geográficas de la subregión Montes de María y la depresión Momposina en el centro de Bolívar, las principales actividades económicas que sustentan a las familias (disfuncionales a falta de padre o madre, o ambos) en la zona la constituyen la pesca y las labores agropecuarias. Dadas las condiciones climáticas sufridas en la zona en los últimos años, dichas familias que, aproximadamente en un 90\% son de estratos 1 (DANE, reporte institucional, San Andrés y sistema clasificatorio del SISBEN), el poder adquisitivo ha sido notoriamente disminuido, subsistiendo entonces del desempeño de labores varias tales como oficios informales como el mototaxismo y el cobro de ayudas derivadas de programas sociales gubernamentales, como Familias en Acción.

\subsection{DISEÑO METODOLÓGICO}

Se parte de la intención de medir la incidencia de una variable independiente (Pensamiento computacional) en otra dependiente (resolución creativa de problemas), considerando también la disposición de grupos objetos de estudio previamente conformados. Es decir, no hubo muestreo aleatorizado para estructuración de grupos, denotando con ello que la elección de grupos intactos, la razón por la que surgen y la manera como se formaron es un tema independiente del estudio (Hernández y Fernández, 2006). En este sentido, se opta por la implementación de un diseño cuasi-experimental con dos grupos, experimental y control, con pre y pos-test y control de equivalencia estadística. El grupo seleccionado como experimental estaba representado por los estudiantes de grado octavo, grupo 1, debido al carácter unitario del grupo en la I. E. Rafael Núñez, se da la necesidad de requerir de otro grupo para que cumpliese funciones del tipo control, y este lo representaron estudiantes de octavo dos de la I. E. Luis Villafañe, en otra localidad corregimental.

En la siguiente tabla (tabla 1) se relacionan datos demográficos de los grupos. 
Tabla 1. Datos demográficos de los grupos objetos de estudio.

\begin{tabular}{|c|c|c|c|c|c|}
\hline Grupo & Rol & Institución & \# Estudiantes & Promedio edad & \% Género \\
\hline $8^{\circ} 1$ & Experimental & Rafael Núñez & 27 & 14,33 & M: 48,14 F:51,85 \\
\hline $8^{\circ} 2$ & Control & Luis Villafañe & 25 & 14,40 & M:44,00 F:56,00 \\
\hline & & & Total: 52 & & \\
\hline
\end{tabular}

Fuente: elaboración propia.

\subsection{INSTRUMENTO DE EVALUACIÓN}

El test 2PCS es uno de los principales productos de esta investigación. La denominación que atañe a este instrumento se deriva de las iniciales sustraídas de la funcionalidad de este instrumento: "Pensamiento computacional (PC) y Solución creativa de problemas (SCP, del inglés). Su objetivo es el de permitir determinar los desempeños de los estudiantes en los grupos experimental y Control, objetos de investigación. Este test fue sometido a validación por pares, pruebas estadísticas (Alfa Cronbach) para medir fiabilidad por medio de pilotajes, y fue aplicado a los diferentes grupos respetando la igualdad de condiciones ambientales, de horarios y contextuales. También se sometió a ligeros cambios de redacción, de estructura, sin alterar su esencia. Esto con el objeto de evitar sesgos relacionados con la maduración y la familiarización de los estudiantes con el instrumento en los distintos momentos de aplicación (Pre-test y Pos-test).

Este instrumento está fundamentado en un compendio de referenciación teórica derivada de la revisión y estudio de literatura desde el análisis epistemológico y funcional de las variables de este estudio. Con todo ello, la intención es la medición de habilidades y conceptos referidos al pensamiento computacional y la forma como estos son requeridos e implementados al momento de resolver problemas desde la capacidad creativa de los estudiantes, asumidas estas habilidades como componentes del proceso creativo (García, 1998). Este instrumento propone una situación inicial contextualizada, la cual dadas sus características propositivas, retadoras y atractivas, se constituye como mecanismo metafórico problémico (Gros, 1997) que estructura y guía la intención didáctica de este recurso.

Inicialmente, el diseño parte de un prototipo compuesto de 16 ítems, y fue sometido a un proceso de pilotaje con el objeto de establecer confiabilidad y consistencia en el instrumento. Las técnicas estadísticas aplicadas implementadas con este fin determinaron la eliminación de cinco ítems, a partir de un coeficiente de Alfa de Cronbach correspondiente a 0.803 , que garantiza la fiabilidad de la escala para el instrumento. En lo referido al aspecto computacional, el test se remite a conceptos fundamentales de la computación y la programación de computadoras teles como variables, iteración, condicionales, ciclos descritos por Joyanes (1996), y la manera como involucra estos conceptos con el mundo real, sugiere su pertinencia y alta significancia, aspectos soportados por la manera en que los estudiantes lo asumieron y resolvieron. 


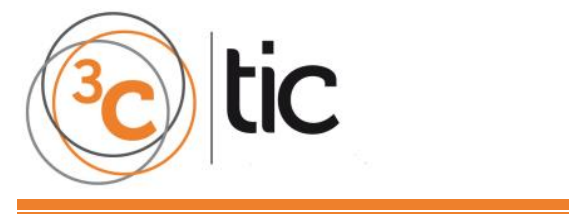

Al final, tras el proceso de pilotaje y consecución la validez y confiabilidad, el test 2PCS queda compuesto por 11 ítems basados en situaciones metafóricas contextualizadas que representan desafíos para el estudiante, envolviéndolo en un rol resolutor y guiado por conceptos computacionales básicos como la iteración, manejo de variables, ciclos y condicionales, a partir de los cuales se estructura cognitivamente la abstracción, como habilidad del pensamiento base en la resolución de problemas y la computación (Wing, 2006).

Como se mencionó en líneas anteriores, la esencia didáctica de este instrumento radica en la posibilidad de sugerir retos al estudiante por medio de situaciones basadas en metáforas problémicas contextualizadas que sugieren la posibilidad de atar emocionalmente y luego ofrecerle la posibilidad de convertirse en artífice de soluciones que exigidas en las problemáticas. Este estilo de mediación está basado en las pruebas aplicas por PISA, como las liberadas de la edición 2003 en el ámbito de solución de problemas, entre las que se destacan situaciones como el sistema de canales de riego, el campamento, el congelador, sistema de transporte y diseño por computadora (INECSE, 2003).

De manera que la situación problema del test 2PCS, en la edición pre test, producto de todo este abordaje, se constituye así:

\section{EL NECIO RATÓN}

"Carlos y Maria son hermanos, ellos saben que en su casa hay un necio ratón, pero nunca lo
han visto. Están seguros de que solo hay un ratón que todas las noches llega hasta el mesón de
la cocina en busca de migajas para comer. Carlos piensa que es un ratón muy grande (con
más de cuatro centimetros de alto, sabiendo que los ratones normalmente llegan máximo a los
6 centimetros de altura), pero Maria piensa que es un ratón muy pequeño (dos o menos
centimetros de alto)".
Cierto día, los hermanos deciden emplear un plan para alimentar al ratón y determinar sí éste
es grande o pequeño. Para ello los hermanos consiguieron una caja totalmente sellada, sín
huecos ni aberturas; y un bisturí con el que se puede cortar cartón con facilidad.

Ilustración 1. Problema del test 3PCS.

Fuente: Adaptación de López, 2013.

Una manera de proponer retos a partir de la anterior situación la constituye el siguiente ítem de muestra, en el cual se parte de una Figura de flujo, una técnica gráfica básica para diseño de algoritmos (Joyanes, 1996) y luego se propone la elección de la opción, previo análisis, comprensión y proposición.

La siguiente ilustración describe tal propuesta:

Ítem relacionado: 
7) En el Diagrama 2 están ubicados unos círculos rojos, los cuales pueden entenderse como:

a) Material o sustancia con lo cual se puede capturar la huella del ratón y asi saber hasta donde llegó, y luegoo determinar su tamaño.

b) Trozos de madera que se usan para hacer creer al ratón que es comida y lograr que siga buscando hasta encontrar el verdadero queso, con lo que puede determinarse que el ratón es pequeño sino aparece el queso, o grande en caso contrario.

c) Pedazos de queso ubicados con la intención de determinar un tamaño muy aproximado al real del ratón.

d) Una mezcla de las ideas a) y b) es lo más adecuado.

Ilustración 2. Problema del test 3PCS.

Fuente: Adaptación de López, 2013.

A resolver de acuerdo a la Figura 1:

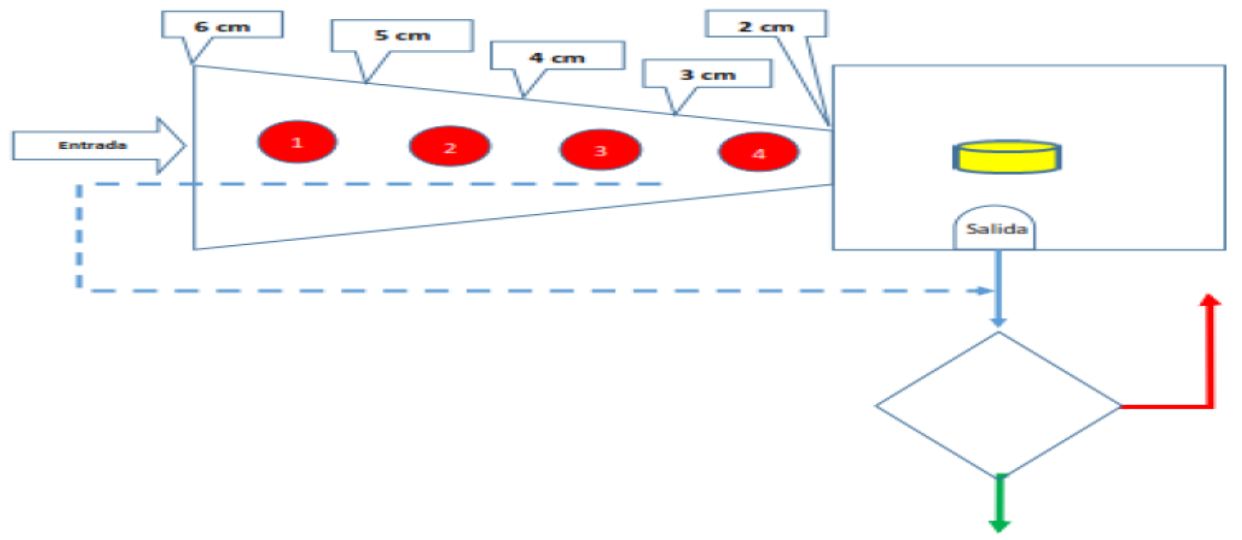

Figura 1. Figura de flujo con modelo de solución alternativo.

Fuente: elaboración propia.

\subsection{PROGRAMA DE INTERVENCIÓN PEDAGÓGICA}

La intervención pedagógica proyectada para propiciar la adquisición de competencias de pensamiento computacional y encaminada al desarrollo potencial de habilidades creativas para la solución de problemas por parte de los estudiantes es una inspiración derivada de diversos aportes teóricos de la pedagogía y la computación. Se refierieren especialmente a aportes de Biggs (2005) y Pólya (1945), quienes de acuerdo a la naturaleza didáctica de sus contribuciones permiten la estructuración de un diseño instruccional de ingentes privilegios formativos. Estos fundamentos teóricos fueron abordados y dispuestos metodológicamente con el objetivo de estructurar una didáctica que permitiese abordar los aspectos conceptuales fundamentales del pensamiento computacional, enmarcado en la disciplina de la programación de computadoras. Esto sucede al tiempo que se transfieren a los componentes educativos correspondientes por medio de la mediación con el ambiente de iniciación en la programación Scratch y orientado hacia el potenciamiento de habilidades en resolución de problemas.

Esta intención pedagógica se caracteriza fundamentalmente por la constitución de climas de formación agradables y creativos, logrando por parte de los estudiantes la asimilación de 
contenidos y el desarrollo de habilidades cognitivas para su aplicación en contextos reales mediante la asignación del rol construccionista al cual se refiere Pappert (1970). Este rol es propiciado básicamente a partir de las posibilidades que por medio de la programación tendrá que ser capaz de diseñar el estudiante y construir sus propios videojuegos, asumidos estos desde la tipología "Arcade", por el hecho de que sus características principales son un diseño sencillo y controles fáciles de asimilar, niveles cortos y de dificultad ascendente y una interrupción mínima entre niveles. Estas características se usaban inicialmente con el objeto de mantener a los jugadores en acción por un corto tiempo, de manera que pronto tuvieron la necesidad de cumplir con las exigencias de desempeño en juego para poder tener la posibilidad de volver a desafiar a la máquina. Estas características sencillas posibilitan y coadyuvan a la intención pedagógica de la intervención. Entre los principales juegos clásicos referenciados se destacan El Arkanoid, también conocido comúnmente con el nombre de Pong, con un jugador o Player, carreras de autos donde se tiene que esquivar obstáculos.

El diseñar y programar un juego para computadora, aspecto que atañe a esta investigación, implica disponer de habilidades computacionales de considerable nivel cognitivo, de conocimientos básicos que se deben implementar en un entorno de programación, en este caso Scratch, bajo ciertas condiciones técnicas. De manera que este aspecto se constituye como el mecanismo mediacional de ingentes privilegios, con el que se motiva la instrucción del estudiante una vez son conscientes de que para lograr tal producto deben conocer, aprender y desarrollar las capacidades de abstracción y metacognición que posibilite sus implementaciones en diversas situaciones y contextos. Un ejemplo de ello es el aprendizaje del concepto de variable, en computación es algo básico que resulta necesario en la construcción de cualquier software. De manera que el estudiante debe antes conocer conceptual y funcionalmente este aspecto básico y luego disponer de la capacidad de implementarlo en su obra, por ejemplo para el control de vidas, conteo de errores o control de puntaje. Este rol construccionista es el que sustenta y orienta el desarrollo de las habilidades pretendidas con la intervención pedagógica.

\subsection{DESCRIPCIÓN DEL PROCESO DE INTERVENCIÓN}

El diseño del proceso de intervención se constituirá de tres fases basadas en enfoques computacionales para el diseño genérico de algoritmos, designadas así:

- Fase 1 (Entrada): Esta fase, inicialmente da cabida a una caracterización de los estudiantes por medio de un test de representación favorito (VAK), con el cual se puede obtener una idea muy cercana de con qué medios pueden llegar a aprender de manera preferible. Tras este paso previo, se procede a acercar al estudiante al por qué aprender lo que se pretende enseñarles, haciendo uso de material audiovisual que dan muestra de casos, experiencias y situaciones que representan en ellos una manera motivadora hacia el aprendizaje, en cuanto dichos recursos se acercan a su vida cotidiana, su entorno y constituyen modelos a seguir. Charlas informales acompañaran estas sesiones con el fin de conocer mejor los intereses y necesidades de los estudiantes. En consecuencia, esta fase básicamente genera los espacios para 
que los estudiantes se familiaricen con términos computacionales del ámbito de la programación, comprendan sus fines y adquieran un acercamiento a los fundamentos de las técnicas de diseño de algoritmos (Figuras de Flujo de Datos y Pseudocódigos). También aquí se conseguirá la familiarización del estudiante con el entorno software de programación Scratch, sus potencialidades y mecanismos de funcionamiento, para que luego puedan llegar al momento de adquisición del conocimiento de los conceptos elementales de la programación y la lógica computacional.

Esta fase comprenderá un espacio de tiempo de 18 horas presenciales en tres semanas.

- Fase 2 (Procesamiento): Está constituida por momentos o sesiones en las que se arman los escenarios por los cuales los estudiantes implementan las funcionalidades de los conceptos y herramientas adquiridos en la "Fase 1". Consiste en llevar a cabo proyectos Scratch con temáticas relacionadas con el entorno real, en los que requieran la selección, implementación y valoración del funcionamiento de las herramientas Scratch tomadas en cuenta por los estudiantes.

En este segundo gran momento se dará capacitación respecto a la utilización de técnicas, estrategias y métodos guiados por el construccionismo y las orientaciones de pensamiento creativo como proceso, sin dejar de lado la sensibilización, la transferencia de conocimientos ni la elaboración de propuestas concretas de solución a situaciones predefinidas. Todo esto con el fin de que los estudiantes adquieran conocimientos y habilidades en la utilización de mecanismos apoyados en la lógica computacional para resolver problemáticas relacionas con el mundo real. Es el momento de interacción verdadera con las situaciones a resolver, con la computadora y el software especializado, y paralelamente se dispone de feedbacks como concepto clave de retroalimentación para guiar de manera óptima el desarrollo cognitivo y metacognitivo. Todo esto apoyado en los compañeros y en el docente, con el fin de refinar cada vez más la madurez cognitiva, el aprendizaje colaborativo y, en algunos casos, el trabajo en equipo.

Esta fase constituye un espacio de tiempo de 24 horas en cuatro semanas.

- Fase 3 (Salida): En esta fase, después de haber adquirido los "suministros" con las habilidades cognitivas requeridas para diseñar soluciones algorítmicas básicas mediadas por computadora empleando el entorno Scratch, los estudiantes se encuentran en la capacidad de implementar soluciones a situaciones reales con el mencionado software, permitiéndose así, comprender con mayor facilidad los mecanismos de funcionamiento de sistemas naturales y artificiales, lo que conlleva a proponer soluciones creativas en las que se requieren la funcionalidad de estos.

En este espacio de tiempo, después de transfer los conocimientos y habilidades logradas en el ámbito netamente curricular, los estudiantes protagonizarán expresiones construccionistas que darán cuenta del potencial pedagógico del 


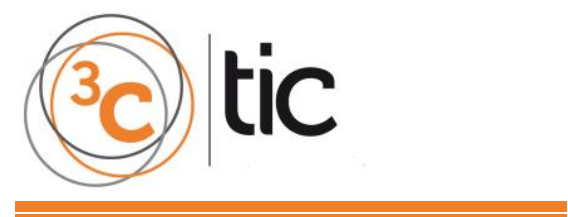

pensamiento computacional para la producción cognitiva en relación a la solución creativa de problemas. Aquí el estudiante demuestra haber adquirido las habilidades pretendidas con dicha sesión y las aplica a situaciones que guardan relación con su entorno y susceptibles de modelización en Scratch.

El espacio de tiempo reservado para esta fase es de 18 horas presenciales por tres semanas.

La siguiente tabla (Tabla 2) describe en brevemente este proceso de intervención:

Tabla 2. Estructura de intervención pedagógica.

\begin{tabular}{|c|c|c|c|}
\hline Identificación & Nivel SOLO & Descripción & Duración H/S. \\
\hline $\begin{array}{l}\text { ENTRADA } \\
\text { Fase 1/Pronóstico }\end{array}$ & Preestructural & $\begin{array}{l}\text { Motivación inicial e identificación } \\
\text { de y familiarización. Se procura la } \\
\text { adquisición del conocimiento } \\
\text { desde perspectivas conceptuales } \\
\text { elementales, las cuales los } \\
\text { estudiantes aún no relacionan } \\
\text { con potenciales mecanismos de } \\
\text { entendimiento y solución de } \\
\text { situaciones. }\end{array}$ & 18 horas / 3 semanas. \\
\hline \multirow{2}{*}{$\begin{array}{l}\text { PROCESAMIENTO } \\
\text { Fase 2/ Proceso }\end{array}$} & Uniestructural & \multirow{2}{*}{$\begin{array}{l}\text { Profundización del conocimiento } \\
\text { con apoyo del docente y de los } \\
\text { pares (compañeros de clases). }\end{array}$} & \multirow[t]{2}{*}{24 horas / 4 semanas. } \\
\hline & Multiesructural & & \\
\hline \multirow{3}{*}{$\begin{array}{l}\text { SALIDA } \\
\text { Fase 3/ Producto }\end{array}$} & Relacional & \multirow{3}{*}{$\begin{array}{l}\text { Creación de conocimiento basado } \\
\text { en el apoyo mínimo por parte de } \\
\text { pares, demostrando mayor } \\
\text { autonomía y habilidades } \\
\text { Metacognitivas por parte del } \\
\text { estudiante. }\end{array}$} & \multirow[t]{3}{*}{ 18horas / 3 semanas. } \\
\hline & & & \\
\hline & Abstracta ampliada & & \\
\hline
\end{tabular}

Fuente: adaptada de Biggs (2005).

\section{RESULTADOS}

Partiendo de la secuencialidad del proceso metodológico investigativo de diseño cuasiexperimental con dos grupos, uno experimental y otro control, con implementación de pre prueba - pos prueba y control de equivalencia, por lo que surgen las siguientes etapas de análisis de resultados, apoyada en procedimientos estadísticos descriptivos y probabilísticos, aspectos relevantes concernientes a la información registrada y los resultados obtenidos:

\section{Etapa 1: presentación y análisis inter grupos (pre prueba) del proceso de intervención}

En esta etapa se lleva a cabo un análisis para determinar las características de los grupos Experimental y Control por medio del test "2PCS", diseñado para tal fin. Este test fue 


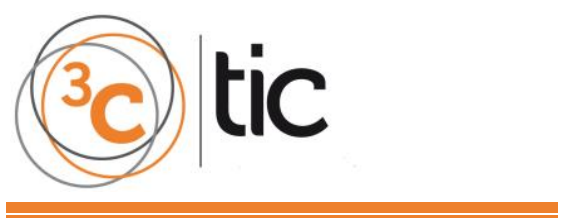

aplicado a los diferentes grupos respetando la igualdad de condiciones ambientales, de horarios y contextuales.

El fin primordial perseguido con la aplicación del test, en modo pre prueba, fue comprobar si los grupos objeto de estudio poseían características que los hiciesen estadísticamente equivalentes desde el punto de vista de habilidades computaciones y de resolución de problemas. En el sentido en que se trata de dos grupos distintos, se realiza para ellos un tratamiento estadístico como muestras independientes aunque bajo tratamiento de igualdad de condiciones.

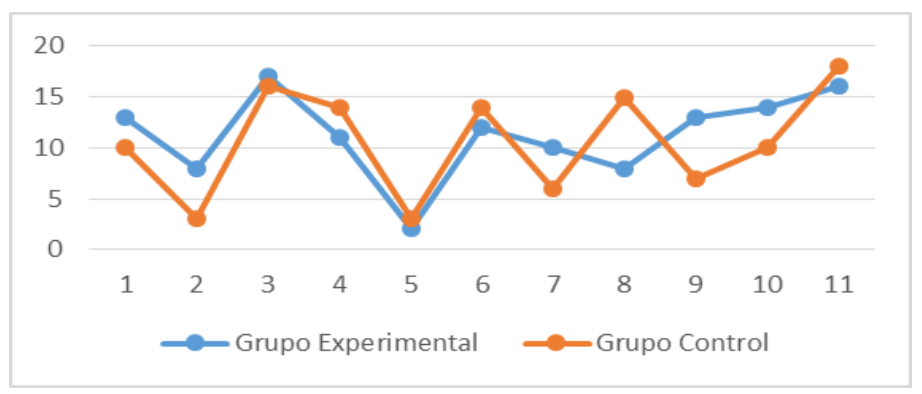

Figura 2. Comparación de resultados según ítems correctamente contestados por los grupos Experimental y Control. Fuente: elaboración propia.

Siguiendo en la misma línea descriptiva, se lleva a cabo una comparación de los promedios de aciertos de los estudiantes en cada grupo, para lo cual se tiene que el grupo Experimental registra un 4,59 y el grupo Control un 4,64. Vista esta información desde una representación porcentual tenemos $41,75 \%$ y $42,18 \%$ para Experimental y Control, respectivamente.

Si bien hasta el momento se ha dejado en clara la equivalencia de los grupos, previamente a una intervención pedagógica en el marco investigativo, y este mismo marco es importante anotar la caracterización cognitiva de ambos grupos, en el sentido que las competencias abordadas registran niveles bajos: $41,75 \%$ Grupo experimental y 42,18\% Grupo Control. Estos resultados sometidos a una conversión de escalas de desempeño no logran llegar al nivel básico. El promedio de aciertos del grupo Experimental equivale según la escala institucional "1-5" (PEI, IE Rafael Núñez - IE Luis Villafañe) correspondería de manera general a aun desempaño "Bajo"; igual situación para el grupo Control.

La siguiente tabla (tabla 3) detalla la indeseable situación de los bajos niveles de los estudiantes en las habilidades medidas con el test.

Tabla 3. Porcentaje de desempeños en escala de valoración institucional.

\begin{tabular}{|l|l|l|l|l|}
\hline Grupos / Porcentaje de desempeños & BJ & B & A & S \\
\hline Grupo Experimental & $92,6 \%$ & $7,4 \%$ & $0 \%$ & $0 \%$ \\
\hline Grupo Control & $94 \%$ & $6 \%$ & $0 \%$ & $0 \%$ \\
\hline
\end{tabular}

Fuente: elaboración propia. 


\subsection{ANÁLISIS ESTADÍSTICO A PARTIR DE LA PRUEBA U DE MANN WHITNEY}

Tras realizar el análisis de los grupos desde la estadística descriptiva se procede con la aplicación de la prueba no paramétrica de Mann Whitney, con el objeto de determinar o no diferencias entre los resultados de la aplicación de la pre-prueba "2pcs" en ambos grupos, y corroborar entonces que son equivalentes. Esto con el propósito de establecer criterios de aprobación o rechazo de la hipótesis central de investigación.

Este análisis comparativo inter-grupos arrojó resultados $(Z=-0,009$ y $p$-valor=0,992) a partir de los cuales es posible inferir que entre los grupos, Experimental y Control, no existen diferencias estadísticamente significativas, en el sentido que el valor " $p$ " obtenido sobrepasa notoriamente el nivel de significancia 0,005 (5\%), dando lugar a la aprobación de la hipótesis nula (Ho), y determinando por tanto que las muestras son equivalentes.

Tabla 4. Estadístico de contraste de U de Mann-Whitney.

\begin{tabular}{|c|c|c|c|c|}
\hline \multicolumn{5}{|c|}{ Rangos } \\
\hline & Grupo & $\mathrm{N}$ & Rango promedio & Suma de rangos \\
\hline \multirow[t]{3}{*}{ AciertosPC } & 1 & 27 & 26,52 & 716,00 \\
\hline & 2 & 25 & 26,48 & 662,00 \\
\hline & Total & 52 & & \\
\hline \multicolumn{3}{|c|}{ Estadísticos de contraste ${ }^{a}$} & & \\
\hline & & AciertosPC & & \\
\hline \multicolumn{2}{|c|}{ U de Mann-Whitney } & 337,000 & & \\
\hline \multicolumn{2}{|c|}{ W de Wilcoxon } & 662,000 & & \\
\hline \multicolumn{2}{|c|}{$\mathbf{Z}$} &,- 009 & & \\
\hline \multicolumn{2}{|c|}{ Sig. asintót. (bilateral) } & 992 & & \\
\hline \multicolumn{3}{|c|}{ a. Variable de agrupación: Grupo } & & \\
\hline
\end{tabular}

Fuente: Análisis SPSS v21. BD del estudio.

En síntesis, con esta primera etapa del análisis de resultados se logró establecer que en el momento de enfrentarse a la pre-prueba "2pcs" los estudiantes del grupo control y el grupo experimental poseían características iniciales similares en relación con los niveles desempeño de habilidades relacionadas con el pensamiento computacional y de resolución de problemas.

\section{Etapa 2: Presentación y análisis intra grupo (grupo control)}

En esta etapa del proceso se lleva a cabo un estudio estadístico intra grupal, tomando como referencia los resultados de la pre-prueba en el grupo Control y los resultados de la Posprueba en el mismo grupo, lo que le da un tratamiento de muestras relacionadas al método estadístico a aplicar. Obviamente, según el modo de operación y la información requerida para el procedimiento los resultados son referidos al mismo grupo pero en diferentes momentos, después de llevar a cabo una implementación didáctica. Tras finalizarlo, se procede según la misma lógica empleada en la anterior etapa. 
La siguiente figura (Figura 3) expone los resultados del pre-test y Pos-test en el grupo control que permite apreciar que entre uno y el otro no hay diferencias significativas, aunque la amplitud de la onda que registra la línea de resultados del Pos-test indica una mínima disminución en el desempeño respecto a la pre-prueba. Todo esto debido a que el pos-test, aunque abordaba las mismas temáticas y el mismo formato didáctico, se tornaba un tanto más complejo debido a la naturaleza de la situación problema, la cual varió su formulación respecto de la primera.

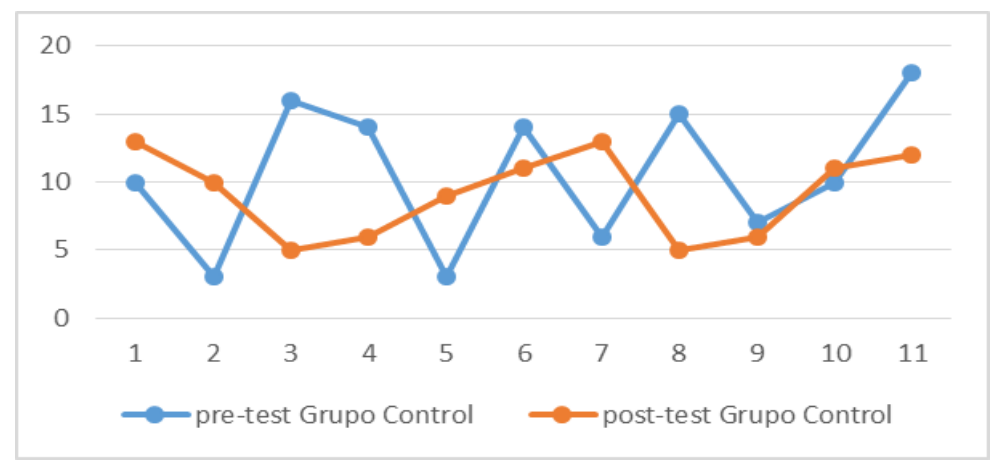

Figura 3. Comparación de resultados según ítems correctamente contestados por el grupo Control durante pre-test y Pos-test. Fuente: elaboración propia.

Como otro mecanismo de soporte estadístico descriptivo para corroborar la relación estrecha entre los resultados de la pre-prueba y la Pos-pruebe en el grupo Control, se referencia la tabla 5, que contiene los promedios de aciertos en las dos pruebas: $4,64(42,18 \%)$ y $4,04(36,72 \%)$, respectivamente y según orden de aplicación de las pruebas. En esta tabla se registra una disminución en el desempeño grupal en la Pos-prueba, aun así, las diferencias no se constituyen como significativas, sugiriendo que los resultados de las pruebas son similares.

Tabla 5. Promedios y porcentajes de aciertos en la pre-prueba y pos-prueba, grupo control.

\begin{tabular}{|l|c|c|}
\hline & Promedio de aciertos & Porcentaje de aciertos \\
\hline Grupo Control (Pre-prueba) & 4,64 & $42,18 \%$ \\
\hline Grupo Control (Pos-prueba) & 4,04 & $36,72 \%$ \\
\hline
\end{tabular}

Fuente: elaboración propia.

Como último elemento descriptivo de esta etapa, se lleva a cabo la conversión de los resultados hacia la escala de valoración de los sistemas de evaluación de las instituciones a las que pertenecen los grupos. La siguiente tabla (Tabla 6) describe los resultados de este proceso en la etapa de análisis intra-grupo, caso específico del grupo Control.

Tabla 6. Porcentaje de desempeños en escala de valoración institucional. Resultados pre-prueba/Posprueba Grupo Control.

\begin{tabular}{|l|l|l|l|l|}
\hline Porcentaje de desempeños & BJ & B & A & S \\
\hline Grupo Control (Pre-prueba) & $94 \%$ & $6 \%$ & $0 \%$ & $0 \%$ \\
\hline Grupo Control (Pos-prueba) & $92 \%$ & $8 \%$ & $0 \%$ & $0 \%$ \\
\hline
\end{tabular}

Fuente: elaboración propia. 


\subsection{ANÁLISIS ESTADÍSTICO A PARTIR DE LA PRUEBA DE WILCOXON}

Con esta prueba de Wilcoxon se pretende establecer si entre estas dos muestras de resultados existen o no diferencias estadísticamente significativas, por medio de lo cual se niega o aprueba la hipótesis nula, basado en la comparación intra-grupo de los resultados de la aplicación de la pre-prueba y Pos-prueba en el grupo Control, en este caso. Por medio de este procedimiento es posible concebir, en términos concretos, si se presentaron avances o no en el desempeño de los resultados obtenidos por los estudiantes del grupo Control, lo que en realidad determina la significatividad en la relación de las muestras de resultados.

Al aplicar la prueba al conjunto de resultados se obtienen los valores $Z=-1,211$ y $p=0,226$. El siguiente es el reporte a partir de software especializado.

Tabla 7. Estadístico de contraste Wilcoxon, grupo control.

\begin{tabular}{|c|c|c|c|c|}
\hline \multicolumn{5}{|c|}{ Rangos } \\
\hline & & $\mathrm{N}$ & Rango promedio & $\begin{array}{l}\text { Suma de } \\
\text { rangos }\end{array}$ \\
\hline \multirow{4}{*}{$\begin{array}{l}\text { PosPrueba } \\
\text { PrePrueba }\end{array}$} & Rangos negativos & $14^{\mathrm{a}}$ & 11,68 & 163,50 \\
\hline & Rangos positivos & $8^{b}$ & 11,19 & 89,50 \\
\hline & Empates & $3^{c}$ & & \\
\hline & Total & 25 & & \\
\hline \multicolumn{5}{|c|}{ a. PosPrueba < PrePrueba } \\
\hline \multicolumn{5}{|c|}{ b. PosPrueba $>$ PrePrueba } \\
\hline \multicolumn{5}{|c|}{ c. PosPrueba $=$ PrePrueba } \\
\hline \multicolumn{5}{|c|}{ Estadísticos de contraste $^{a}$} \\
\hline & \multicolumn{4}{|c|}{ PosPrueba - PrePrueba } \\
\hline Z & \multicolumn{4}{|l|}{$-1,211^{b}$} \\
\hline $\begin{array}{ll}\text { Sig. } & \text { asintót. } \\
\text { (bilateral) } & \end{array}$ & \multicolumn{4}{|l|}{,226 } \\
\hline \multicolumn{5}{|c|}{ a. Prueba de los rangos con signo de Wilcoxon } \\
\hline \multicolumn{5}{|c|}{ b. Basado en los rangos positivos. } \\
\hline
\end{tabular}

Fuente: Análisis SPSS v21. BD del estudio.

Esta información permite inferir que el p-valor o nivel de significancia obtenido con la prueba de Wilcoxon corresponde a 0,226, el cual es mayor que el nivel de significación de 0.05 (5\%). Por medio de este dato se puede establecer que el resultado de la Pos-prueba de los estudiantes del grupo Control no difiere con los resultados de la pre-prueba con relación al mismo grupo, es decir, no hay diferencias estadísticamente significativas entre la pre-prueba y la Pos-prueba para esta muestra. En este sentido, es posible aseverar que los estudiantes del grupo control al presentar la Pos-prueba obtienen un desempeño similar al obtenido en la pre-prueba, pues no presentaron avances en las habilidades computacionales y de resolución de problemas. Todo esto, pese a que por medio de los métodos estadísticos abordados inicialmente en esta etapa se evidenció una disminución en los desempeños, determinando que los resultados no fueron estáticos, no obstante, tal variación negativa no 


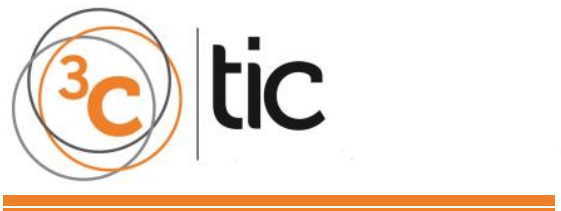

significó una razón para determinar que las muestras relacionadas no eran similares. Muestra de ello es el valor de significancia obtenido.

\section{Etapa 3: presentación y análisis intra grupo (grupo experimental)}

La particularidad de esta etapa radica en que, debido a que se lleva a cabo una comparación de muestras relacionadas de resultados de un grupo obtenidos previamente a un proceso de intervención pedagógica (pre-prueba), y Poserior a este (Pos-prueba). Dicho de otro modo, se pretende establecer si tal proceso pedagógico planeado y dispuesto para el desarrollo cognitivo de las determinadas habilidades surge el efecto esperado. De ser así, se establecerá que las muestras relacionadas guardan diferencias estadísticamente significativas.

A continuación, se presentan los datos correspondientes a la aplicación de los 11 ítems del test "2PCS" en sus versiones pre-test y Pos-test.

En el siguiente gráfico de líneas se presentan los resultados del pre - test y Pos - test en el grupo Experimental, permitiendo apreciar que entre los resultados de los grupos muestrales de resultados, antes y después, se presentan diferencias significativas. Así, se da soporte a la interpretación de que el aumento de los desempeños en la pos-prueba respecto de los de la Pos - prueba representan un importante aumento, y se infiere por tanto (desde métodos descriptivos) la existencia de diferencias significativas desde esta perspectiva intra-grupal Experimental.

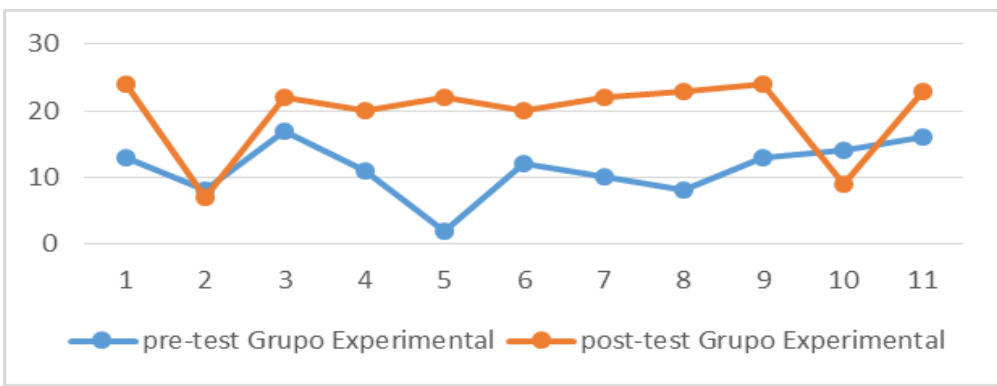

Figura 4. Grupo experimental en pre prueba y pos prueba.

Fuente: elaboración propia.

A continuación, puede observarse de manera general el porcentaje de aciertos en las respuestas por parte de los estudiantes en la pre-prueba $(4,59)$, contrastado con el promedio de aciertos de éstos en la pos-prueba, posterior al proceso de intervención pedagógica.

Tabla 8. Promedios y porcentajes de aciertos en la pre-prueba y Pos-prueba por parte del grupo Experimental.

\begin{tabular}{|l|c|c|c|c|}
\hline & \multicolumn{2}{|l|}{ Promedio de aciertos } & \multicolumn{2}{l|}{ Porcentaje de aciertos } \\
\hline Grupo Experimental (Pre-prueba) & 4,59 & \multicolumn{2}{|c|}{$41,75 \%$} \\
\hline Grupo Experimental (Pos-prueba) & 8 & B & A & S \\
\hline Porcentaje de desempeños & BJ & $7,4 \%$ & $0 \%$ & $0 \%$ \\
\hline Grupo Experimental (Pre-prueba) & $92,6 \%$ & $44,4 \%$ & $29,6 \%$ & $11,1 \%$ \\
\hline Grupo Experimental (Pos-prueba) & $14,8 \%$ & 4 & \\
\hline
\end{tabular}

Fuente: elaboración propia. 


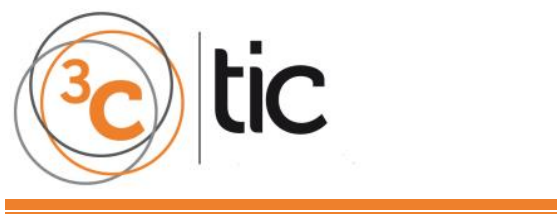

En la tabla inmediatamente anterior (Tabla 8) es posible evidenciar el mejoramiento en los niveles de desempeño en la aplicación de la Pos-prueba, registrando además desempeños en los niveles "Alto" y "Superior". Se logra una disminución considerable en el nivel "Bajo", de $92,6 \%$ a 14,8\%, y los resultados en el nivel "Básico" aumentan notoriamente. Esta información viene a demostrarnos que existen diferencias significativas en los resultados de la pre-prueba y la Pos-prueba, lo cual será confirmado más adelante con la prueba no paramétrica.

\subsection{ANÁLISIS ESTADÍSTICO A PARTIR DE LA PRUEBA DE WILCOXON}

Los resultados de la prueba de Wilcoxon aplicada al conjunto de resultados de las pruebas arrojó los siguientes valores: $\mathrm{Z}=-4.471$ y $\mathrm{p}=0,000$.

Tabla 9. Estadístico de contraste Wilcoxon, grupo experimental. Fuente: Análisis SPSS V21. BD del estudio.

\begin{tabular}{|c|c|c|c|c|}
\hline \multicolumn{5}{|c|}{ Rangos } \\
\hline & & $\mathrm{N}$ & Rango promedio & $\begin{array}{l}\text { Suma de } \\
\text { rangos }\end{array}$ \\
\hline \multirow{4}{*}{$\begin{array}{l}\text { PosPrueba } \\
\text { PrePrueba }\end{array}$} & Rangos negativos & $0^{\mathrm{a}}$ & ,00 & ,00 \\
\hline & Rangos positivos & $26^{b}$ & 13,50 & 351,00 \\
\hline & Empates & $1^{\mathrm{c}}$ & & \\
\hline & Total & 27 & & \\
\hline \multicolumn{5}{|c|}{ a. PosPrueba $<$ PrePrueba } \\
\hline \multicolumn{5}{|c|}{ b. PosPrueba > PrePrueba } \\
\hline \multicolumn{5}{|c|}{ c. PosPrueba $=$ PrePrueba } \\
\hline \multicolumn{3}{|c|}{ Estadísticos de contraste ${ }^{a}$} & & \\
\hline & \multicolumn{2}{|l|}{ PosPrueba-PrePrueba } & & \\
\hline Z & $-4,471^{b}$ & & & \\
\hline $\begin{array}{ll}\text { Sig. } & \text { asintót. } \\
\text { (bilateral) } & \end{array}$ & 000 & & & \\
\hline \multicolumn{3}{|c|}{ a. Prueba de los rangos con signo de Wilcoxon } & & \\
\hline \multicolumn{3}{|c|}{ b. Basado en los rangos negativos. } & & \\
\hline
\end{tabular}

Fuente: elaboración propia.

El p-valor obtenido con la prueba Wilcoxon corresponde a 0,000, el cual es evidentemente menor a 0,005 (5\%), aceptando por tanto la hipótesis de que entre los resultados de la preprueba y pos-prueba existen diferencias significativas, en el sentido que los resultados de esta última difieren positivamente respecto de los de la primera. Se sugiere, por tanto, que los deseables desempeños de los estudiantes en la Pos-prueba se constituyen como situación resultante de la puesta en marcha de la intervención pedagógica basada en el desarrollo del pensamiento computacional para potenciar habilidades en la resolución creativa de problemas. 


\section{Etapa 4: Presentación y análisis inter grupo (Pos-prueba grupo experimental / grupo control)}

En esta última etapa del proceso de presentación y análisis de los resultados se focaliza el estudio de los datos provenientes de la aplicación de la Pos-prueba a los estudiantes del grupo experimental, con el fin de medir los desempeños de las habilidades obtenidas tras ser instruidos por medio del proceso de intervención pedagógica fundada en el desarrollo del pensamiento computacional. A partir de ello, y al igual que en las anteriores etapas, se pretende el hallazgo de diferencias estadísticamente significativas, con relación a los resultados derivados de la aplicación de la Pos-prueba a los estudiantes del grupo Control, la cual se llevó bajo principios de igualdad de condiciones como en las otras pruebas en este proceso investigativo.

En consecuencia, se pretende un análisis inter-grupo, con muestras independientes, cuya intención es contrastar los resultados de dos muestras distintas, tomadas en grupos de estudiantes diferentes, pero bajo condiciones similares.

A continuación, se exhiben los resultados del pos-test en el grupo experimental y en el grupo control, por medio de los cuales se evidencia que los resultados revelan un alto grado de diferencias significativas en los resultados del grupo Experimental con respecto a los resultados del grupo Control.

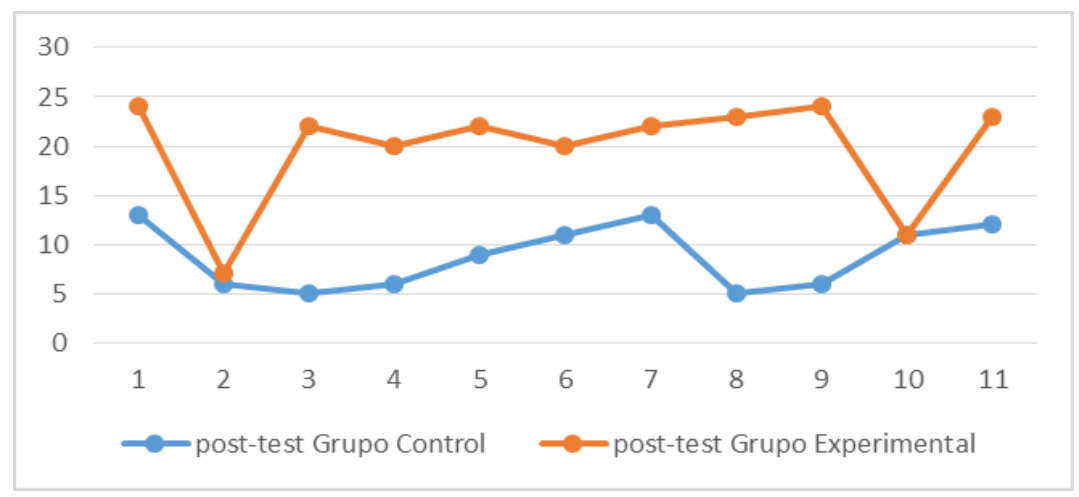

Figura 5. Resultados pos - test en los grupos de estudio.

Fuente: elaboración propia.

Más allá de la situación de los ítems " 2 " y "10", que ambos grupos de resultados se constituyeron en elementos de cierto nivel resolutorio, según lo observado en este gráfico, es notable que los resultados obtenidos muestran un incremento en los desempeños a favor del grupo Experimental, lo que prueba que el desempeño superior que se obtuvo no fue por casualidad o por el proceso de maduración, sino que obedece a la incidencia del plan de intervención realizada, pues fue en este grupo con quienes se implementó.

La tabla 10 muestra de forma general el promedio de aciertos por parte de los estudiantes y el porcentaje de los mismos. Mediante estos datos se evidencia que el promedio del grupo Experimental $(4,04)$ supera al representado por el grupo Control (8). Quedando, por tanto, demostrada la existencia de diferencias significativas entre los grupos muestrales tras la intervención. 
Los porcentajes de los promedios ilustran desde otra perspectiva esta situación. El porcentaje representado por el grupo intervenido es de un $72,72 \%$, contra un $36,72 \%$ del grupo no sometido a intervención. Proyectando una diferencia de $36,00 \%$ entre los dos grupos de resultados.

Tabla 10. Promedios y porcentajes de aciertos en la Pos-prueba, grupo control y experimental.

\begin{tabular}{|c|c|c|c|c|}
\hline & \multicolumn{2}{|c|}{ Promedio de aciertos } & \multicolumn{2}{|c|}{ Porcentaje de aciertos } \\
\hline Grupo control (Pos-prueba) & \multicolumn{2}{|c|}{4,04} & \multicolumn{2}{|c|}{$36,72 \%$} \\
\hline $\begin{array}{l}\text { Grupo } \\
\text { prueba) }\end{array}$ & \multicolumn{2}{|l|}{8} & \multicolumn{2}{|l|}{$72,72 \%$} \\
\hline Porcentaje de desempeños & BJ & B & A & $\mathbf{S}$ \\
\hline Grupo Control (Pos-prueba) & $92 \%$ & $8 \%$ & $0 \%$ & $0 \%$ \\
\hline Grupo Experimental (Pos-prueba) & $14,8 \%$ & $44,4 \%$ & $29,6 \%$ & $11,1 \%$ \\
\hline
\end{tabular}

Fuente: elaboración propia.

\subsection{ANÁLISIS ESTADÍSTICO A PARTIR DE LA PRUEBA U DE MANN WHITNEY}

Con el fin de demostrar que entre los dos grupos de estudio (grupo control y grupo experimental) existen diferencias estadísticamente significativas, se recurre a un análisis desde la estadística no paramétrica, en específico la prueba $U$ de Mann Whitney. Se realiza con la finalidad de establecer el valor de significancia, mediante la cual se constate la existencia de tales diferencias desde los niveles de desempeño, justo después de un plan pedagógico aplica al grupo Experimental.

De la aplicación de esta prueba estadística al total de resultados obtenidos por ambos grupos en la Pos-prueba, se obtiene que $Z=-5,734$ y $p$-valor $=0,000$.

Tabla 11. Estadístico de contraste. Grupo Experimental y Control (Pos-prueba).

\begin{tabular}{|c|c|c|c|c|}
\hline \multicolumn{5}{|c|}{ Rangos } \\
\hline & Grupo & $\mathrm{N}$ & $\begin{array}{l}\text { Rango } \\
\text { promedio }\end{array}$ & Suma de rangos \\
\hline \multirow[t]{3}{*}{ AciertosPC } & 1 & 27 & 38,00 & 1026,00 \\
\hline & 2 & 25 & 14,08 & 352,00 \\
\hline & Total & 52 & & \\
\hline \multicolumn{3}{|c|}{ Estadísticos de contraste $^{a}$} & & \\
\hline & \multicolumn{2}{|c|}{ AciertosPC } & & \\
\hline U de Mann-Whitney & \multicolumn{2}{|c|}{27,000} & & \\
\hline W de Wilcoxon & \multicolumn{2}{|c|}{352,000} & & \\
\hline Z & \multicolumn{2}{|c|}{$-5,734$} & & \\
\hline $\begin{array}{ll}\text { Sig. } & \text { asintót } \\
\text { (bilateral) }\end{array}$ & \multicolumn{2}{|c|}{,000 } & & \\
\hline \multicolumn{3}{|c|}{ Variable de agrupación: Grupo } & & \\
\hline
\end{tabular}

Fuente: Reporte de análisis SPSS v21, BD del proyecto. 
El valor de significancia obtenido, 0.000, obviamente no supera el nivel de significancia 0.005 (5\%), con lo cual se rechaza la hipótesis nula. Es decir, la distribución total no es la misma entre los dos grupos, lo que conduce a inferir que los avances en los desempeños de los estudiantes fueron producto de los tratamientos y no del hecho de enfrentarse nuevamente a una prueba similar, dando lugar a aspectos relacionados con procesos normales de maduración. Dicho de otro modo, las diferencias significativas entre los resultados del Posprueba en el grupo Experimental, con respecto a los resultados de esta misma prueba en el grupo control, dan fe de la efectividad de la intervención pedagógica implementada con el primer grupo referido.

En este sentido, se da lugar a la aceptación de la hipótesis alternativa de investigación: "Existen diferencias estadísticamente significativas en el desarrollo de competencias relacionadas con la resolución creativa de problemas luego que estas antes y después de ser potencializadas por medio del desarrollo de habilidades del pensamiento computacional en los estudiantes de $8^{\circ}$ de la institución educativa Rafael Núñez de San Andrés - Bolívar".

\subsection{ANÁLISIS CUALITATIVO DEL PROCESO DE INTERVENCIÓN}

Una vez se les planteó el desarrollo de una investigación a los estudiantes de grado $8^{\circ}$ de la institución educativa Rafael Núñez de San Andrés Bolívar, tomaron la noticia sin mayores signos de efusividad pero tampoco con expresiones de incomodidad ni desacuerdo, a pesar de que tendrían que emplear parte de su tiempo que excedía su intensidad horaria académica habitual. Sin embargo, el rol experimental de dicho grupo, una vez conocieron de las características del entorno a virtual a conocer y sus potencialidades en lo referente a creaciones propias, el interés aumentó y su disposición fue totalmente favorable. Tras algunas demostraciones, videos y testimonios de sus pares en otros lugares del mundo, dispuestos en el sitio web oficial de Scratch: Scratch.mit.edu.

A partir de esta experiencia, los estudiantes demostraron su potencial y afinidad para el desarrollo de competencias digitales, relacionadas en este caso específico con las ciencias computacionales, dejando por tanto en evidencia el acierto metodológico de la propuesta pedagógica de intervención. El soporte de lo anteriormente expuesto lo representa un material audiovisual constituido por 14 archivos digitales, los cuales son parte de la evidencia del proceso de investigación, y a partir de lo cual os estudiantes objeto de estudio dan fe de su provecho formativo.

Durante las 20 sesiones llevadas a cabo, los 27 estudiantes se mostraron muy entusiastas participando como aprendices de un conocimiento que facilita la interacción con las nuevas tecnologías, las cuales sirven, entre otros aspectos, como apoyo para el aprendizaje de las diferentes áreas del conocimiento. Una entrevista no estructurada realizada verbalmente a determinados estudiantes durante ciertos apartes del proceso, sobre todo cerca del final de éste, posibilitó el registro de opiniones, percepciones y pareceres que estos tenían con referencia al proceso investigativo del cual fueron partícipes. De este proceso de auscultación podemos destacar algunas apartes muy interesantes. 
Uno de los mecanismo mediacionales tomados en cuenta durante el proyecto lo constituyó el diseño e implementación del clásico juego arcade conocido como Pac-man, el cual referencia innegable en la historia de los videojuegos. La estudiante autora revela detalles de cómo llevó a cabo su proyecto y expresa su satisfacción con el proceso. Tras la petición del docente investigador: "bueno trata de mostrarnos que fue lo que hiciste en el código", la estudiante responde: "bueno...este...la idea era crear el pac-man...y que cuando el tocara las uvas o las frutas él se las comiera, y que cuando, o sea tocará estos obstáculos - señalaba la pantalla del computador- iniciaba de nuevo, y...tratara de llegar a la llegada, que en este caso es el cuadrito rojo este que está aquí -volvía a señalar-". iLlegada! exclama el profesor. La estudiante continúa explicando: "...exactamente, cuando él lo toca se esconde, significa que ya llego, y ya".

Más adelante, la misma estudiante mientras explica el código por bloques del videojuego, detalla el proceso de implementación de variables: "ehh... para que estas se escondan, agregué un <<por siempre sí>> para que si tocaban al pac-man estás se escondían. Y también creé una variable con el fin de que estas contaran cuántas uvas o cuántas naranjas se comía el pac-man".

"iOk, muy bien muy bien!", exclama el profesor. Y pide: "...juega una última vez y ya". $<<$ Risas $>$

Otro estudiante también detalló su proyecto Arkanoid con rivales “...pues yo intenté poner aquí con el color, que si toca la barra que rebote y mover 10 pasos... para cuando también tocara los ladrillos rebotara y los ladrillos se escondieran. Agregué sonidos y una bandera para que el darle clic todo volviera a su posición". El joven se despide cómicamente. $<<$ Risas >> Dice su nombre y termina exclamado "Yo trabajo con Scratch". <<Risas >>.

Otra niña, a la pregunta del profesor: ¿Qué te ha parecido la experiencia con Scratch hasta ahora? Responde: "Eh... muy buena ya que nos divertimos mucho, podemos explotar nuestras capacidades y pa'que es muy bacano".

El $100 \%$ de las manifestaciones por parte de los estudiantes fueron positivas, todos expresaban su parecer a su manera, entre timidez, otros un poco más abiertos y espontáneos, y otros preferían hacerlos fuera de cámaras.

\section{DISCUSIÓN}

Resulta coherente partir de una alusión a Mitra (2005), quien por medio de su experimento "Hole in the wall", determinó, entre otros aspectos de considerable validez, que las computadoras constituyen per se como un mecanismo con el cual se puede asegurar el aprendizaje dadas ciertas circunstancias contextuales y disposiciones didácticas. Lo que sugeriría que el ir más allá del simple uso de herramientas computarizadas para procurar emplear el poder computacional de estas para la promoción de ambientes de instrucción guiada en los que se priorice la creación de artefactos computacionales, la posibilidad de 
modelar y simular situaciones del mundo real, representaría un escenario pedagógico de ingentes dimensiones.

En este sentido, resulta válido y pertinente la intención de esta investigación al concebir la resolución de problemas como una vía apropiada para la potenciación de habilidades resolutivas y creativas en los estudiantes. Esto es debido a que la idea no es pretender comunicar conocimientos de forma acabada, sino que desde la misma dinámica, desarrollo y generación de soluciones se establecen dinámicas sinérgicamente estructuradas basadas en los intereses, necesidades, saberes y sentires de los estudiantes (Ortiz, 2009). Desde lo cual, el pensamiento computacional se constituye como mecanismo mediacional pertinente para el desarrollo de habilidades del pensamiento, destacadas entre ellas las relacionadas con la resolución de problemas, en el sentido que sus implicaciones derivan potencialidades pedagógicas soportadas en los aportes de Wing (2006), quien considera el PC como un proceso que conlleva habilidades relacionadas con "la solución de problemas, el diseño de sistemas y la comprensión de la conducta humana, haciendo uso de conceptos fundamentales de la informática. El pensamiento computacional incluye una serie de herramientas mentales que reflejan la amplitud del campo de la informática" (p.33).

Desde aquí, resulta pertinente la idea de concebir al Pensamiento Computacional como un proceso de pensamiento humano estructurado para la resolución de problemas que incluye una serie de características y disposiciones cognitivas y técnicas. Las habilidades de un pensador computacional van más allá de la capacidad de la apropiación técnica y uso de diversas herramientas, programas o sistemas software, o de programar una computadora, en el estricto sentido técnico. En realidad, el concebir un pensador de esta índole implica asociarlo con habilidades para afrontar, entender y resolver problemas con mayor eficacia, a partir de estructurados niveles de abstracción, orientados a la construcción de soluciones en diversos ámbitos de la vida y en cualquier área del conocimiento.

Esta postura concuerda con lo propuesto por Brennan y Resnick (2012), quienes conciben tres dimensiones asociadas al pensamiento computacional: conceptos, prácticas y perspectivas computacionales. Éstas, en un sentido práctico, son el producto de experiencias basadas en el lenguaje de programación visual Scratch, como un entorno que permite a los estudiantes crear juegos o simulaciones empleando los conceptos de programación de una forma más creativa y divertida. Desde esta óptica, se privilegia el desarrollo del pensamiento computacional, la disposición para afrontar y resolver problemas, usando conceptos computacionales por medio de ambientes favorables como Scratch, que garantizan estos avances cognitivos sin la necesidad de asumir directamente el abordaje de conceptos de la computación de alta exigencia cognitiva.

Wing, (2006) sostiene que la esencia del pensamiento computacional es la abstracción y que las abstracciones para la computación son las herramientas "mentales" y las computadoras las herramientas "metálicas" que automatizan las abstracciones. Estas habilidades son entendidas esencialmente para el desarrollo de aplicaciones informáticas, para el desarrollo de software, pero también puede ser utilizado para apoyar la resolución de problemas en todas en las distintas disciplinas, incluyendo las humanidades, matemáticas y la ciencia. De 
manera que el incorporar este tipo de contenidos y técnicas en ámbitos escolares representa un gran potencial pedagógico, en el sentido que los estudiantes que aprenden PC a través del currículo pueden empezar a ver una relación entre las ciencias dispuestas en la academia, así como entre la vida dentro y fuera del aula (Rizzi, 2016).

Por otra parte, Resnick (2013), durante su participación en el evento TED, se refiere a los jóvenes de hoy como nativos digitales y considera que esta denominación puede conllevar confusión. En el sentido, hoy día la mayor parte de los jóvenes son básicamente consumidores de tecnología, no productores. Son usuarios expertos, pero no son creadores. En un mismo contexto formativo, la situación se asemejaría a la de estudiantes que saben leer pero no saben escribir. De manera que se reafirma entonces la idea de formar a los alumnos en ciencias de la computación les dotará de las habilidades necesarias para utilizar plenamente las tecnologías de la era digital.

Estas oportunidades de aprendizaje llevadas a experimento con la presenta investigación lograron establecer como resultado primordial la validez y pertinencia de los ocho aspectos que Lawicki (2015) considera como razones ineludibles del saber codificar:

De forma resumida:

1. Aprender a codificar nos enseña muchas lecciones vitales.

2. Aprender a codificar nos enseña sobre aprender y enseñar.

3. Codificar ayuda a pensar y a resolver problemas cuando se programa.

4. Todas las asignaturas escolares adquieren sentido y guardan relación con el mundo exterior para el estudiante de ciencias de la computación.

5. La codificación amplía la creatividad.

6. Al aprender ciencias computacionales nos preparamos a nosotros mismos para el éxito.

7. Con las ciencias de la computación podemos cambiar el mundo.

8. Al programar parece que tengamos superpoderes.

9. Por tanto, al final la idea no es pretender que los estudiantes en el futuro se conviertan en programadores profesionales, sino, el promover el hecho de que aprender a programar ofrece beneficios cognitivos de ingentes dimensiones desde el sentido del potenciamiento de la creatividad. Ésta se ve promovida a partir del pensamiento lógico, conllevando por ende a la formación de verdaderas competencias digitales que conducen al entendimiento del funcionamiento de las nuevas tecnologías y sus posibilidades de mejoramiento de su relación con diversos ámbitos de la vida diaria.

\section{CONCLUSIONES}

Partiendo de las condiciones estadísticas de los resultados, es posible determinar que la intervención pedagógica diseñada según parámetros metodológicos coherentes con el desarrollo del Pensamiento Computacional permitió el desarrollo habilidades del pensamiento relacionadas con la resolución creativa de problemas en estudiantes de básica secundaria. 
Desde este contexto resulta conveniente considerar este potencial desde diferentes ópticas, asumidas desde el impacto de este estudio, tenemos:

- Los mecanismos de implementación de las tecnologías de la información y comunicación en contextos educativos de nuestra región Caribe poseen un aspecto desfavorable en común, en el sentido que son concebidos como una herramienta novedosa, pero al momento de implementarlas se prescinde de referentes y soportes metodológicos que procuren por un fructífero uso en los escenarios educativos de nuestro contexto.

- El abordaje de los procesos formativos concernientes al área de Tecnología e Informática adeuda cambios de los paradigmas convencionales improductivos, lo que se evidencia desde la subutilización de las herramientas en función de las potencialidades creativas y propositivas de los estudiantes.

- Según las disposiciones de la actual era digital y la sociedad del conocimiento resulta necesario concebir el Pensamiento Computacional desde una perspectiva integral en función del aprendizaje de otras disciplinas o áreas del conocimiento.

- La resolución creativa de problemas como mecanismo potenciador de aprendizaje, implica aspectos variados relacionados con metodologías, disposiciones contextuales, características cognitivas y emocionales de los estudiantes, de manera que se constituye como un complejo constructo cargado de potencial didáctico para el desarrollo integral de los educandos.

- El diseño e implementación sistemática de una metodología de intervención pedagógica, apoyada en referentes teóricos cognitivistas como el construccionismo de Papert y el modelo de alineación constructivista de Biggs, apoyados en los aportes del modelo de Polya, el cual a su vez estuvo orientado por conceptos y técnicas propios de las ciencias computacionales, se constituye como garantía para potenciar el desarrollo de la competencia resolución de problemas de la vida diaria desde el potencial creativo de los estudiantes.

- Se establece un avance a nivel investigativo en lo concerniente a la implementación de las ciencias computacionales en contextos escolarizados, suscitando la premisa de generar pedagogía orientada a la creación y la proposición, prescindiendo con ello de las tendencias habituales orientadas por el carácter consumista de los distintos actores involucrados en los procesos formativos.

- El Test "2PCS" se constituye como un instrumento válido para la medición de habilidades relacionadas con la resolución creativa de problemas. Este test es el principal producto obtenido a partir de esta investigación, la manera como involucra conceptos computacionales - variables, iteración, condicionales, ciclos - y su relación con el mundo real sugiere su pertinencia y alta significancia, aspectos soportados por la manera en que los estudiantes lo asumieron y resolvieron, y por el control de variables extrañas - maduración cognitiva y familiarización - evidenciado en los resultados analizados Posteriormente.

Por último, y desde todo este contexto configurado a partir de las anteriores consideraciones, la presente es un valioso intento por contribuir al fomento de la computación cognitiva para la producción de nuevas innovaciones pedagógicas en contextos de educación escolarizada, promoviendo a manera de recomendación el ser pedagógicamente hábiles para aprovechar las potencialidades de los avances en tecnología para el beneficio de experiencias educativas que optimicen la formación de los estudiantes 
dadas las exigencias de la actual era digital y la urgente sociedad del conocimiento. En este orden de ideas, al integrar la programación de computadoras en los planes de estudio es posible garantizar el desarrollo de habilidades potenciadas y complementadas desde una formación apoyada en la codificación (Balanskat \& Engelhardt, 2015).

Todos estos productos del proceso de investigación cobran para efectos de aspectos relacionados con la Resolución Creativa de Problemas y su privilegiada relación con la programación de computadoras, total coherencia con los planteamientos de Robinson, quien a partir del interrogante "Do School kill Creativity?" sustenta su discurso en la idea de que los sistemas educativos actuales son anacrónicos, es decir, desfasados de los con relación a los contextos y realidades de la actualidad. En este sentido,las naciones necesitan mentes que piensen de forma creativa y entiendan los valores culturales. Al igual que requieren docentes que además de ser capaces de enseñar cosas, también propicien escenarios para que los estudiantes logren cultivar su talento (Robinson, 2007), en pro de una educación tendiente a la capacidad productiva, más que a la reproductiva.

\section{REFERENCIAS BIBLIOGRÁFICAS}

ATEC21S. (2015). 21st Century Skills. Recuperado de: <http://www.atc21s.org/>.

Balanskat, A., \& Engelhardt, K. (2015). Computing our future: Computer programming and coding-priorities, school curricula and initiatives across Europe. European Schoolnet. Recuperado de: $<$ http://fcl.eun.org/documents/10180/14689/Computing+our+future final.pdf/746e 36b1-e1a6-4bf1-8105-ea27c0d2bbe0/>.

Biggs, J. (2005). Calidad del aprendizaje universitario: Cómo aprenden los estudiantes.

Brennan, K., \& Resnick, M. (2012). New Frameworks for Studying and Assessing the Development of Computational Thinking. Proceedings of the 2012 annual meeting of the American Educational Research Association, Vancouver, Canada.

García García, J. (1998). La creatividad y la resolución de problemas como bases de un modelo didáctico alternativo. Revista Educación y Pedagogía, 10(21), 145-174.

Hernández Sampieri, R., Fernández Collado, C., \& Baptista Lucio, P. (2010). Metodología de la investigación (5a. ed.). México: McGraw-Hill.

Lawicki, J. (2015). De consumidores de tecnología a agentes del cambio. Recuperado de: $<$ https://innovacioneducativa.fundaciontelefonica.com/blog/2016/06/29/deconsumidores-de-tecnologia-a-agentes-del-cambio/>.

López, L. (2013). ¿Por qué trabajar la programación de computadoras en la escuela? Preguntas, sugerencias y herramientas para el aula, 1(6), 9-11. Recuperado de $<$ https://www.usfq.edu.ec/publicaciones/para el aula/Documents/para el aula 06 L0004 para el aula 06.pdf/>. 
López, J. C. (2014). ¿Por qué es importante promover que los estudiantes desarrollen el pensamiento computacional? En: Mirada Relpe, reflexiones iberoamericanas sobre las TIC en educación, 78-83. Buenos Aires: Relpe.

Mir, B. (2009). La competencia digital, competencia metodológica. Presentation, CCCB Montalegre, 5 08001-Barcelona.

Mitra, S. (2013). Build a School in the Cloud, TEDx. Archivo de vídeo. Recuperado de: $<$ https://www.youtube.com/watch?v=y3jYVe1RGaU/>.

Newell, A. \& Simon, H. (1972). Human Problem Solving. Englewood Cliffs, NJ: Prentice-Hall.

Papert, S. \& Harel, I. (2002). Situar el Construccionismo. Traducción del Centro Latinoamericano para la Competitividad y el Desarrollo Sostenible (INCAE). Recuperado de: <http://web.media.mit.edu/ calla/ Readings/situar el construcionismo.pdf/>.

Polya, G. (1945). How to Solve It. Garden City, New York: Doubleday.

Resnick, M. (2007). Cultivando las semillas para una sociedad más creativa. Act. Inv. En Educ., 8(1). DOI:10.15517/aie.v8i1.9306.

Resnick, M., Silverman, B., Kafai, Y., Maloney, J., Monroy-Hernández, A., \& Rusk, N. et al. (2009). Scratch: Programming for All. Commun. ACM, 52(11), 60. DOI:10.1145/1592761.1592779.

Rizzi, C. 2016. Ciencias de la Computación en Ciencias. Curso Mooc. Google. Recuperado de: $<$ https://cs4hs-ccec.appspot.com/ccec/course/>.

Robinson, K. (2007). Do School kill Creativity?, TEDx. Archivo de vídeo. Recuperado de: $<$ https://www.youtube.com/watch?v=iG9CE55wbtY/>.

Wing, J. (2006). Computational thinking. Communications Of The ACM, 49(3), 33. DOI:10.1145/1118178.1118215. 